\title{
J. HARTMANIS \\ On effective speed-up and long proofs of trivial theorems in formal theories
}

Revue française d'automatique, informatique, recherche opérationnelle. Informatique théorique, tome 10, $\mathrm{n}^{\circ} \mathrm{R} 1$ (1976), p. 29-38.

<http://www.numdam.org/item?id=ITA_1976_10_1_29_0>

(C) AFCET, 1976, tous droits réservés.

L'accès aux archives de la revue « Revue française d'automatique, informatique, recherche opérationnelle. Informatique théorique » implique l'accord avec les conditions générales d'utilisation (http://www.numdam.org/legal. php). Toute utilisation commerciale ou impression systématique est constitutive d'une infraction pénale. Toute copie ou impression de ce fichier doit contenir la présente mention de copyright.

\section{Numdam}

Article numérisé dans le cadre du programme

Numérisation de documents anciens mathématiques

http://www.numdam.org/ 
R.A.I.R.O. Informatique Théorique

(vol. 10, $\mathrm{n}^{\circ} 3$, mars 1976, p. 29 à 38)

\title{
ON EFFECTIVE SPEED-UP \\ AND LONG PROOFS OF TRIVIAL THEOREMS \\ IN FORMAL THEORIES $\left({ }^{*}\right)\left({ }^{1}\right)$
}

\author{
by J. Hartmanis $\left({ }^{2}\right)$ \\ Communicated by G. AUSIELLO
}

\begin{abstract}
In this note we give a very simple proof which shows that in many interesting formal mathematical theories, axiomatizable as well as decidable ones, for every given formalization we can effectively find infinite subsets of trivially true theorems which require as long proofs in the given formalism as the hardest theorems of the theory. Thus showing that for these theories every formalism is doomed to be blind to the triviality of infinite sets of theorems, which can be found effectively. Furthermore, it follows that for all (sufficiently, large) constructable tape and time bounds there exist sets whose recognition can be effectively speeded up on infinite subsets and that such sets appear naturally. Thus showing that for many concrete problems every algorithm can be effectively sped-up on infinite subsets.
\end{abstract}

\section{INFORMAL INTRODUCTION}

The main purpose of this note is to give a simple proof which shows that in many interesting mathematical theories for every formal (mechanical) proof method, there exists infinite sets of "trivially true" theorems which require as long proofs in the given formalism as the hardest theorems. Surprisingly, some of these infinite sets of trivially true theorems with long proofs, can be found effectively for every given proof procedure and thus the proof procedure can be replaced by another one which shortens the proofs of infinitely many theorems without increasing the length of proofs for any others. For related work, see $[2,8]$.

These results hold for many axiomatizable as well as decidable mathematical theories. They also show that there exist recursive functions and recursive sets for which every algorithm computing them can be effectively speeded-up infinitely often. Furthermore, these recursive functions appear naturally, and we observe that specific combinatorial problems fall in the class of problems for which every algorithm can be effectively speeded up. Thus for many computations there do not exist optimal algorithms and every given one can be effectively speeded up infinitely often.

(*) Reçu septembre 1975.

( ${ }^{1}$ This research has been supported in part by National Science Foundation Grants GJ-33171 X and DCR 75-09433.

(2) Department of Computer Science Cornell University, Ithaca.

Revue Française d'Automatique, Informatique et Recherche Opérationnelle $\mathrm{n}^{\circ}$ mars 1976 
These results show very clearly that we pay a price for formalizing mathematics. In every formalization, infinite sets of trivial theorems will require very long proofs. Thus giving a very dramatic and quantitative explanation why we should not and in practice do not freeze a formalism when discussing or doing mathematics. It also gives a warning that a necessarily long proof in a formal system does not certify that the result is non-trivial.

These observations are particularly interesting in view of the recent results about decision complexity of decidable mathematical theories, which show that many of these theories, though decidable, are not practically decidable. For example, it has been shown that for the Pressburger Arithmetic, the first order theory of natural numbers under addition, any proof procedure is such that the length of the proofs cannot be bounded by a double exponential in the length of the theorems [3]. Some even more dramatic complexity results are known about other decidable theories [8]. Our observation adds the postscript, that not only are these theories very hard to decide, but that every decision procedure requires as much work (long proofs) as the hardest theorems in the theory on infinite sets of trivially true theorems and that from the description of the decision procedure, we can effectively obtain such infinite sets of trivially true theorems with long proofs. For related observations see also [8].

It is interesting to compare these results with the Blum Speed-up Theorem [1]. We recall that Blum proved that for every recursive function $g$ there exist recursive functions $f$ for which any algorithm can be speeded up by the function $g$ almost everywhere. Unfortunately, even if a function $f$ has an almost everywhere speed-up, the faster algorithms cannot be found effectively from a given algorithm, nor could one effectively compute from which point on the speed-up started. Finally, there is a wide spread belief that functions with an almost everywhere speed-up are artificially constructed and that they do not appear naturally in mathematics or practical computing.

The situation with the functions, problems and mathematical theories which have speed-up infinitely often is quite different. This speed-up is effective and we can explicitely show subsets on which computations can be sped-up. Furthermore, we do not have to construct artificial examples to show existence of such speed-up. The most important mathematical theories have for every formalization proof-shortening infinitely often, and many combinatorial problems which we are interested in solving are such that every algorithm can be sped-up infinitely often. As a matter of fact, mathematical theories and sets which have speed-up infinitely often appear to be more natural than those which do not have such speed-up. See also $[4,6]$.

Finally, it should be pointed out that it is not at all clear whether we can reap any practical benefits from the fact that for certain specific problems every algorithm for their solution will work unnecessarily hard on an infinite 
set of simple cases of this problem. We can always find such an infinite set for these problems effectively from the description of the algorithm, but it may turn out that they do not appear often enough in practice to justify replacing the given algorithm by an improved one which we know can never be optimal.

\section{SPEED-UP FOR CREATIVE SETS}

It is well known that the provable theorems of many important mathematical theories form creative sets [7]. One such mathematical theory is Peano Arithmetic, which is based on Peano's axioms with those instances of the Peano induction axiom that can be expressed in elementary arithmetic. Several other well known examples can be easily found, and it is clear that the creative sets form a very important class of sets in mathematics [7].

We recall that a set $A, A \subseteq \Sigma^{*}$, is creative iff $A$ is recursively enumerable and there exists a recursive function $\sigma$ such that for every Turing machine $M_{i}$, which enumerate a subset of $\bar{A}=\Sigma^{*}-A$, that is $T\left(M_{i}\right) \subseteq \bar{A}, \sigma(i)$ is in $\bar{A}-T\left(M_{i}\right)$. We can also define equivalently, a recursively enumerable set $A$ to be creative iff every other recursively enumerable set $B$ can be one-one reduced to $A$, i. e. there exists a one-one recursive function $f$ such that $w$ is in $B$ iff $f(w)$ is in $A$. From these definitions it easily follows that all creative sets are recursively isomorphic, i. e., if $A$ and $C$ are creative sets, $A \subseteq \Sigma^{*}$ and $B \subseteq \Gamma^{*}$, then there exists a recursive bijection $f: \Sigma^{*} \rightarrow \Gamma^{*}$ such that $w \in A$ iff $f(w) \in B$. Thus, but for a recursive translation, all creative sets are the same, and as it will be seen, our results apply to all of them.

In the first part of this paper we will study the difficulty of recognizing creative sets or equivalently the difficulty of proving theorems in mathematical theories, like the Peano Arithmetic. A natural measure of the difficulty of proving a theorem in a formal mathematical theory is the length of the shortest proof. Unfortunately, to do this, we have to know explicitely how the theory is axiomatized and what proof procedures are used. To avoid such unnecessary difficulties we will measure the difficulty of recognizing sets (of provable theorems) by the amount of tape used by Turing machines recognizing these sets. Intuitively, this measure can be justified by observing that for any (reasonable) proof procedure, we can design a Turing machine which for any given theorem successively checks all possible proofs of increasing length until it finds a proof of the given theorem (or never halts if the input is not a provable theorem). Clearly, the amount of tape used by this Turing machine is a lower bound for the length of the proof and corresponds, intuitively, directly to the amount of erasable blackboard space needed to verify the proof or present it. 
Consider the set

$$
\begin{aligned}
& L_{0}=\left\{(i, j, w) \mid i, j \in N, w \in \Sigma^{*},\right. \\
& M_{j} \text { or } M_{i} \text { on }(i, j, w) \text { uses a finite amount of tape and, } \\
& \text { either } M_{i} \text { does not accept }(i, j, w) \\
&\text { or } \left.M_{i} \text { uses more tape than } M_{j} \text { on }(i, j, w)\right\} .
\end{aligned}
$$

Where $i$ and $j$ are binary representations of the corresponding integers and $(i, j, w)$ is the sequence $i, j, w$ enclosed in parentheses. We say that $M_{j}$ uses more than $M_{i}$ on input $x$ if $M_{i}$ uses a finite amount of tape and $M_{j}$ uses at least one more tape square than $M_{i}$ on input $x$.

We now show that the set $L_{0}$ is recursively enumerable. We observe that for any Turing machine $M_{i}$, input $x$ and integer $k$ we can recursively decide whether $M_{i}$ for input $x$ uses more than $k$ tape squares. If $M_{i}$ uses less than $k$ tape squares for input $x$ we can decide whether $x$ is accepted by $M_{i}$ or not. Thus to enumerate $L_{0}$ we just reject all inputs which are not of the form $(i, j, w)$. For input $(i, j, w)$ we check successively for $k=0,1,2, \ldots$ whether $M_{i}$ or $M_{j}$ uses exactly $k$ tape squares for input $(i, j, w)$. If it is found that one of the Turing machines uses a finite amount of tape then we can decide whether $M_{i}$ uses more tape than $M_{j}$; if so $(i, j, w)$ is accepted. If $M_{i}$ uses less tape than $M_{j}$ then we can decide whether $M_{i}$ accepts $(i, j, w)$ and it is accepted iff $M_{i}$ does not accept it.

LEMMA 1 : The set $L_{0}$ is a creative set.

Proof: We know that $L_{0}$ is an r. e. set. To show that it is a creative set let $M_{k^{\prime}}$ be a $T m$ which simulates $M_{k}$ and tests whether $M_{k}$ uses a finite amount of tape; if $M_{k}$ is found to use a finite amount of tape then $M_{k^{\prime}}$ accepts the input if $M_{k}$ does not and vice versa. The function $(k)=\left(k^{\prime}, k^{\prime}, w_{0}\right)$, for any fixed $w_{0}$ in $\Sigma^{*}$, is a productive function for the set $\bar{L}_{0}$, since $T\left(M_{k}\right) \subseteq \bar{L}_{0}$ implies that $\left(k^{\prime}, k^{\prime}, w_{0}\right)$ is in the set $\bar{L}_{0}-T\left(M_{k}\right)$. Thus $L_{0}$ is a creative set, as was to be shown.

THEOREM 2 : Let $M_{i_{0}}$ recognize the set $L_{0}, T\left(M_{i_{0}}\right)=L_{0}$, and let $M_{j_{0}}$ be a Tm which halts for all inputs. Then the regular set

$$
\left\{\left(i_{0}, j_{0}, w\right) \mid w \in \Sigma^{*}\right\}
$$

is a subset of $L_{0}$ and on every input $\left(i_{0}, j_{0}, w\right) M_{i_{0}}$ uses no less tape than $M_{j_{0}}$ on the same input.

Proof : Let $\left(i_{0}, j_{0}, w\right)$ denote the sequence $i_{0}, j_{0}, w$ enclosed in parentheses. Then, for fixed $i_{0}$ and $j_{0}$,

$$
\left\{\left(i_{0}, j_{0}, w\right) \mid w \in \Sigma^{*}\right\}
$$


is a regular set. Since $M_{j_{0}}$ halts for all inputs we see that on every input $\left(i_{0}\right.$, $\left.j_{0}, w\right)$ the Turing machine $M_{i_{0}}$ uses no less tape than $M_{j_{0}}$ on this input. Since otherwise, if $M_{i_{0}}$ accepts $\left(i_{0}, j_{0}, w\right)$ using less tape than $M_{j_{0}}$, we see that $\left(i_{0}, j_{0}, w\right) \in T\left(M_{i_{0}}\right)$ but not in $L_{0}$, contradicting the assumption that $T\left(M_{i_{0}}\right)=L_{0}$; similarly if $M_{i_{0}}$ rejects $\left(i_{0}, j_{0}, w\right)$ using less tape than $M_{j_{0}}$ on this input we have that $\left(i_{0}, j_{0}, w\right) \in L_{0}$ but not in $T\left(M_{i_{0}}\right)$; again a contradiction. If $M_{i_{0}}$ uses no less tape than $M_{j_{0}}$ on $\left(i_{0}, j_{0}, w\right)$ then $M_{i_{0}}$ must accept the input, since othervise we have the contradiction that $\left(i_{0}, j_{0}, w\right)$ is in $L_{0}$ but not in $T\left(M_{i_{0}}\right)$. Thus $\left\{\left(i_{0}, j_{0}, w\right) \mid w \in \Sigma^{*}\right\}$ is a subset of $L_{0}$ and on every element of this subset $M_{i_{0}}$ uses no less tape than $M_{j_{0}}$. This completes the proof.

From this result we immediately get the following.

COROLlARY 3: For every Turing machine which recognizes $L_{0}$ and every recursive function $f$ we can effectively find an infinite regular subset of $L_{0}$ on which for each input of length $n M_{i_{0}}$ uses at least $f(n)$ tape squares for its recognition.

Proof: Immediate from previous theorem. From this result it follows that for every recognizer $M_{i_{0}}$ of $L_{0}$ we can effectively obtain infinite subsets of $L_{0}$ on which $M_{i_{0}}$ uses large amounts of tape but which can be recognized by finite automata, since they are regular subsets.

Considering the members of $L_{0}$ as provable theorems of a mathematical theory, the result asserts that every formalization of this mathematical theory will have infinite sets of "trivially" true theorems but which in the formalism require horrendously long proofs.

To illustrate this, assume that we have a first order mathematical theory with the standard logical connectives and in which we can express such concepts as

$$
\text { " } M_{i} \text { uses less tape than } M_{j} \text { on input } x \text { " }
$$

and

" $M_{i}$ does not accept the input $x . "$

Assume that the theory is strong enough to prove all true statements of the form

" $M_{j}$ uses less tape than $M_{i}$ on input $(i, j, w)$ or $M_{i}$ uses less tape then $M_{j}$ and does not accept the input $(i, j, w) . "$

For example, the Peano Arithmetic is such a mathematical theory.

From our previous result we see that any proof procedure for this mathematical theory will have easily recognizable infinite sets of true theorems such that the length of their shortest proofs in the given formalizm will grow faster than any given recursive function (of the length of the theorems to be proved). 
We conclude this section by observing that these results hold for all creative sets.

CoRollary 4: Let $B$ be a creative set, $M_{i}$ be a Tm which recognizes $B$ and $f$ $a$ recursive function. Then we can effectively find an infinite recursive subset of $B$ which can be recognized by a Tm using $L(n)$ tape but on which the Tm $M_{i}$ uses atleast $f \circ L(n)$ tape.

Proof: This follows from the fact that all creative sets are recursively isomorphic and that the recursive isomorphic function can be computed on a recursively bounded amount of tape.

The family of recursively enumerable sets which have effective speed-up of the type we proved for creative sets has been completely characterized in [2] and Corollary 4 follows from this general characterization, though these proofs are quite complicated.

\section{SUBRECURSIVE ANALOGUES}

Let $L_{i}(n)$ be the maximal number of tape square used by the $T m M_{i}$ on inputs of length $n$. We say that a total function $L(n)$ is tape constructable iff there exists a $\operatorname{Tm} M_{i}$ such that $L(n)=L_{i}(n)$. The following result has been credited to A. Meyer in [8] and is stated there without proof. The auther obtained it independently and only then became aware of its statement in [8].

THEOREM 5: For every tape constructable $L(n) \geqq n$ there exists a recursive set $A_{L}$ (a recursive function $f_{L}: \Sigma^{*} \rightarrow\{0,1\}$ ) such that

a) $A_{L}$ can be recognized on $L(n)$ tape but not on $L_{1}(n)$ tape if

$$
\lim _{n \rightarrow \infty} \frac{L_{1}(n)}{L(n)}=0
$$

b) For every $M_{i_{0}}$ such that $T\left(M_{i_{0}}\right)=A_{L}$ we can effectively find an infinite regular subset $R_{i_{0}}$ of $A_{L}$ such that $M_{i_{0}}$ uses at least $L(n) /\left|M_{i_{0}}\right|$ tape on every member of $R_{i_{0}}$.

Proof: Let $M_{1}, M_{2}, \ldots$ be a standard enumeration of all one-tape $T m$ 's such that the set $\left\{M_{i}\right\}$ is recognizable in polynomial time using linear tape. Let $L(n) \geqq n$ be tape constructable and let

$$
A_{L}=\left\{\left(M_{i}, w\right) \mid M_{i} \text { does not accept }\left(M_{i}, w\right) \text { on } \frac{L\left(\left|\left(M_{i}, w\right)\right|\right)}{\left|M_{i}\right|} \text { tape }\right\},
$$

where $\left|M_{i}\right|$ denotes the length of the description of $M_{i}$. Note that $\left(M_{i}, w\right)$ is in $A_{L}$ if either $M_{i}$ uses more than $\left.L\left(\mid M_{i}, w\right) \mid\right) /\left|M_{i}\right|$ tape or it does not accept the input on this amount of tape. We claim that $A_{L}$ is $L(n)$ tape acceptable by the following method: for input $x$ check if $x$ is of the 
form $\left(M_{i}, w\right)$, if not reject. For input $\left(M_{i}, w\right)$ lay off $\left.L\left(\mid M_{i}, w\right) \mid\right)$ tape and on a separate track of the tape simulate $M_{i}$ on input $\left(M_{i}, w\right)$; accept it if the simulation tries to use more than $L\left(\left|\left(M_{i}, w\right)\right|\right) /\left|M_{i}\right|$ tape, if the simulation halts on the available tape reject it if accepted and vice versa. Clearly, this simulation can be carried out on $L\left(\left|\left(M_{i}, w\right)\right|\right)$ tape and we see that $A_{L}$ is $L(n)$ tape acceptable.

Let $M_{i_{0}}$ be a recognizer of $A_{L}$ and consider the regular set

$$
R_{i_{0}}=\left\{\left(M_{i_{0}}, w\right) \mid w \in \Sigma^{*}\right\} .
$$

The $\operatorname{Tm} M_{i_{0}}$ on input $\left(M_{i_{0}}, w\right)$ either uses more than $L\left(\left|\left(M_{i_{0}}, w\right)\right|\right) /\left|M_{i_{0}}\right|$ tape or it does not. If it does then $\left(M_{i_{0}}, w\right)$ is in $T\left(M_{i_{0}}\right)$. If it does not then either $\left(M_{i_{0}}, w\right)$ is accepted or not. In either case, we get a contradiction: if $\left(M_{i_{0}}, w\right)$ is accepted then $\left(M_{i_{0}}, w\right)$ is in $T\left(M_{i_{0}}\right)$ but not in $A_{L}$ contradict. ing $T\left(M_{i_{0}}\right)=A_{L}$; if $\left(M_{i_{0}} w\right)$ is not accepted then $\left(M_{i_{0}}, w\right)$ is in $A_{L}$ but not in $T\left(M_{i_{0}}\right)$, again contradicting the assumption $T\left(M_{i_{0}}\right)=A_{L}$. Thus we must conclude that $R_{i_{0}} \subseteq A_{L}$ and that on all inputs $\left(M_{i_{0}}, w\right)$ the machine $M_{i_{0}}$ uses more than $L\left(\left|\left(M_{i_{0}}, w\right)\right|\right) /\left|M_{i_{0}}\right|$ tape. From which it also follows that $A_{L}$ is not recognizable on $L_{1}(n)$ tape, provided $\lim _{n \rightarrow \infty}\left[L_{1}(n) / L(n)\right]=0$. This completes the proof.

Next we show that many decidable mathematical theories must have trivial theorems requiring long proofs and that we can effectively find infinite sets of such trivial theorems with long proofs.

Let $T$ be a consistant, decidable mathematical theory, let $L$ and $F$ be tape constructable functions such that for all $n F(n+1)>F(n) \geqq n$ and $L(n) \geqq n$. Let there exist a $\operatorname{Tm} M$ which for each input $\left(M_{i}, w\right)$ writes a formula $\gamma_{i, w}$ in $T$ such that:

a) $\gamma_{i, w}$ is provable in $T$ iff $\left(M_{i}, w\right) \in A_{L}$, that is iff the Turing machine $M_{i}$ does not accept input $\left(M_{i}, w\right)$ on $L\left(\left|\left(M_{i}, w\right)\right|\right) /\left|M_{i}\right|$ tape.

b) $\left|\gamma_{i, w}\right| \leqq F\left(\left|\left(M_{i}, w\right)\right|\right)$ and $M$ use no more than $\left|\gamma_{i, w}\right|$ tape to write $\gamma_{i, w}$ for input $\left(M_{i}, w\right)$.

COROLlaRY 6: Any decision procedure for the theory $T$ requires at least $L \circ F^{-1}(n)$ tape and for every $T m M_{i_{0}}$ which recognizes the theorems of $T$ we can effectively find an infinite subset $T^{\prime}$ of theorems of $T$ such that $T^{\prime}$ is recognizable on linear tape but $M_{i_{0}}$ uses at least c. $L \circ F^{-1}(n)$ tape on every element of $T^{\prime}$, for a constant $c>0$.

Proof: Let $M_{i_{0}}$ recognize the theorems of $T$. We now convert $M_{i_{0}}$ to a recognizer of $A_{L}, M_{i_{00}}$. For input $x M_{i_{00}}$ checks if $x=\left(M_{i}, w\right)$; if not it is rejected. For input $\left(M_{i}, w\right)$ the machine $M_{i_{00}}$ computes $\gamma_{i, w}$ and applies $M_{i_{0}}$ to $\gamma_{i, w}$. Clearly, $M_{i_{00}}$ accepts $A_{L}$. Assume now that $M_{i_{0}}$ operates on $L_{i_{0}}$ tape such that

$$
\lim _{n \rightarrow \infty} \frac{L_{i_{0}}(n)}{L \circ F^{-1}(n)}=0 \text {. }
$$


Then $M_{i_{00}}$ would operate on $L_{i_{0}}[F(n)]$ tape; but then

$$
\lim _{n \rightarrow \infty} \frac{L_{i_{0}}[F(n)]}{L \circ F^{-1} \circ F(n)}=\lim _{n \rightarrow \infty} \frac{L_{i_{0}}[F(n)]}{L(n)}=0,
$$

contradicting the fact that no such recognizer exists for $A_{L}$.

We now show how to obtain the set $T^{\prime}$ using $M_{i_{00}}$. We know that the regular set

$$
R=\left\{\left(M_{i_{00}}, w\right) \mid w \in \Sigma^{*}\right\}
$$

is a subset of $A_{L}$ and that $M_{i_{00}}$ uses at least $L(n) /\left|M_{i_{00}}\right|$ tape on inputs from $R$. We let

$$
T^{\prime}=\left\{\gamma_{i_{00}, \nu} \mid w \in \Sigma^{*}\right\}
$$

clearly, $T^{\prime}$ is an infinite set of true theorems of $T$. To recognize the set $T^{\prime}$ we proceed as follows: for input $\gamma$ (on track below $\gamma$ ) check for $\left(M_{i_{00}}, w\right)$, $\left|\left(M_{i_{00}}, w\right)\right| \leqq|\gamma|$, whether $\gamma=\gamma_{i_{00}, w}$.

If no such $\left(M_{i_{00}}, w\right)$ is found reject the input, otherwise accept it. Clearly, $T^{\prime}$ is recognizable on linear tape, since $\left|\gamma_{i, w}\right| \geqq\left|\left(M_{i}, w\right)\right|$, and the computation of $\gamma_{i, w}$ from $\left(M_{i}, w\right)$ require no more than $\left|\gamma_{i, w}\right|$ tape. On the other hand, since $M_{i_{00}}$ uses $L(n) /\left|M_{i_{00}}\right|$ tape on $R, M_{i_{0}}$ must use at least $L \circ F^{-1}(n) /\left|M_{i_{00}}\right|$ tape, since $\left|\gamma_{i_{00}, w}\right| \leqq F\left(\left|\left(M_{i_{00}}, w\right)\right|\right)$. This completes the proof.

Recent work on the complexity of decision procedures for decidable mathematical theories has shown how statements about Turing machine computations can be efficiently encoded in formulas in these theories so that the statement about the Tm computation is true iff the formula is provable in the theory $[3,8]$. These results can be used not only to establish the complexity of the decision procedures, but also the existance of speed-up for most of these decision procedures [8].

For example, it has been shown [8] that the set

$$
\begin{aligned}
\mathscr{R}=\{R \mid & R \text { is a regular expression } \\
& \quad \text { over } 0,1,(.), \cdot,+, \neg, *, \text { denoting the empty set }\}
\end{aligned}
$$

cannot be recognized on tape bounded by an elementary function. By the same techniques it follows that every $T m$ recognizing this set can be effectively speeded-up by any desired "stack of exponentials" on infinite subsets of $\mathscr{R}$.

We conclude with a look at time complexity of decision procedures and speed-up of proofs for Pressburger Arithmetic [3].

Let $T_{i}(n)$ be the maximal number of steps taken by the $T m M_{i}$ on inputs of length $n$. We say that $T(n)$ is time-constructable iff $T(n)=T_{i}(n)$ for some $M_{i}$. 
COROLlARY 7: Let $T(n) \geqq n^{2}$ be time constructable. Then there exists a set $A_{T}$,

$$
A_{T}=\left\{\left(M_{i}, w\right) \mid M_{i} \text { does not accept }\left(M_{i}, w\right) \text { in time } \frac{T(n)}{\left|M_{i}\right| \log T(n)}\right\},
$$

such that $A_{T}$ is recognizable in time $T(n)$ and if $T\left(M_{i_{0}}\right)=A_{T}$ then the regular set

$$
R=\left\{\left(M_{i_{0}}, w\right) \mid M_{i_{0}} \text { does not accept }\left(M_{i_{0}}, w\right) \text { in time } \frac{T(n)}{\left|M_{i_{0}}\right| \log T(n)}\right\}
$$

is a subset of $A_{T}$ and $M_{i_{0}}$ uses at least

steps for every member of $R$.

$$
\frac{T(n)}{\left|M_{i_{0}}\right| \log T(n)}
$$

Proof: Similar to the proof of Theorem 5. The only difference is in the factor $\log T(n)$ which appears because we have to use a less efficient simulation algorithm than in the tape complexity case [5].

We recall that the Pressburger Arithmetic (PA) is the first order theory of addition of natural numbers [3]. This theory is decidable and it "is one of the simplest, most basic, imaginable mathematical theories" [3]. Furthermore, it has been shown that any non-deterministic $T m$ recognizing the true theorems of PA must use at least time

$$
T(n)=2^{2 c n} \text { for some } c>0 .
$$

From this, one concludes that any "reasonable" proof procedure for PA will have theorems of length $n$ whose shortest proof must be longer than $2^{2^{\text {cn }}}$ [3].

We add to this result the following observation.

THEOREM 8: Let $M_{i_{0}}$ be a Tm which recognizes the theorems of PA. Then we can effectively find an infinite set $T$ of theorems of $\mathrm{PA}$ such that $T$ is recognizable in polynomial time but $M_{i_{0}}$ uses at least time

$$
T(n) \geqq 2^{2 q n}, \text { for some } q>0,
$$

for every element of $T$.

Proof: By a close inspection of the proof techniques used in [3] and using reasoning similar to that in the proof of Corollaries 6 and 7, we can construct the set $T^{\prime}$ of sentences $\gamma_{i_{0}, w}$ in PA asserting that $M_{i_{0}}$ does not accept the input $\left(M_{i_{0}, \text { }}\right)$ in time

$$
\frac{2^{2^{d n}}}{\left|M_{i_{0}}\right| \log 2^{2^{d n}}}=\frac{2^{2^{d n}}}{2^{d n}\left|M_{i_{0}}\right|}, \text { for some } d>0
$$


From this we then conclude that for some $q>0$ and sufficiently large $n$ :

$$
\frac{2^{2^{d n}}}{2^{d n}\left|M_{i_{0}}\right|} \geqq 2^{2^{q n}}
$$

Thus, the recognition of the set $T^{\prime}$ by $M_{i}$ requires at least $22^{q n}$ steps for almost all elements of $T^{\prime}$. By removing the short elements of $T^{\prime}$ we get the desired set $T$. On the other hand, it can be seen from [3] that the set $T$ is recognizable in polynomial time. This completes the proof.

Thus again we see that for this very basic and simple theory we can effectively find, for every proof procedure, infinite sets of theorems which are recognizable as true in polynomial time, but for which the shortest proofs in the formalism are horrendously long. Again pointing out the limitations of formal methods and mechanical proof procedures, even for this very simple mathematical theory.

\section{REFERENCES}

1. M. BLum. A Machine-Independent Theory of the Complexity of Recursive Functions. J. Assoc. Comp. Mach., vol. 14, 1967, p. 322-336.

2. M. Blum and I. Marques. On Complexity Properties of Recursively Enumerable Sets. J. Symbolic Logic, vol. 38, 1973, p. 579-593.

3. M. J. FisCher and M. O. RABIN. Super-Exponential Complexity of Pressburger Arithmetic. In SIAM-AMS Proceedings, vol. 7, p. 27-41. American Math. Soc. Providence, RI, 1974.

4. J. Gill and M. Blum. On Almost Everywhere Complex Recursive Functions. J. Assoc. Comp. Mach., vol. 21, 1974, p. 425-435.

5. J. Hartmanis and J. E. HopCroft. An Overview of the Theory of Computational Complexity. J. Assoc. Comp. Mach., vol. 18, 1971, p. 444-475.

6. N. Lynch. On Reducability to Complex or Sparse Sets. J. Assoc. Comp. Mach., vol. 22, 1975, p. 341-345.

7. H. Rogers. The Theory of Recursive Functions and Effective Computability, McGraw Hill, New York, 1967.

8. L. J. Stockmeyer. The Complexity of Decision Problems in Automata Theory and Logic. Project MAC, Report MAC TR-133, MIT, Cambridge, Mass., July 1974. 